\title{
OSTEOARTICULAR INVOLVEMENT IN CUTANEOS DISSEMINATED SPOROTRICHOSIS: CASE REPORT
}

Débora Rocha de Moura Rodrigues de Aguiar ${ }^{1, *}$, Natasha dos Santos Miranda ${ }^{1}$, Luiz Felipe Dipe Prates Miranda ${ }^{1}$, Carlos Baptista Figueiredo ${ }^{1}$

1. Hospital Federal de Bonsucesso, Rio de Janeiro (RJ), Brazil.

*Corresponding author: deboramouraaguiar@gmail.com

\section{BACKGROUND}

Sporotrichosis is a subcutaneous mycosis, with chronic or subacute manifestation, caused by dimorphic fungi of the genus Sporothrix. Recently, the disease has behaved endemically and, although typical transmission is associated with the management of soil and organic waste, zoonotic transmission has been mainly responsible for the recent epidemiological change in Brazil. In immunocompetent individuals, the evolution of the disease tends to be benign and clinically presents in lymphocutaneous and localized cutaneous forms. However, immunosuppressed, diabetic or alcoholic individuals may present more severe forms with a pattern of cutaneous-disseminated or extracutaneous involvement, which the most common is osteoarticular. Infectious arthritis is more common than osteomyelitis. The most frequently affected joints are the knee, elbow, wrist and ankle.

CASE REPORT

A 63-year-old, hypertensive, decompensated diabetic, and vasculopath patient, diagnosed with cutaneous-disseminated sporotrichosis, was hospitalized due to refractory oral treatment with itraconazole. During admission exam, in addition to the cutaneous lesions spread on the face, trunk and arms, patient presents with edema, hyperemia, pain and limited mobility in the 2nd and 3rd fingers of the right hand (Fig. 1). The patient underwent imaging exams that showed cortical erosion, reduction of joint space and lytic areas in the distal metadiaphyseal region of the ulna, radius and head of the proximal phalanx of the second finger. A bone fragment biopsy was performed on the 2 nd finger and skin biopsy of a lesion on the forehead that showed a granulomatous inflammatory process compatible with sporotrichosis. The proximity between skin lesion and osteoarticular involvement suggests dissemination by contiguity. Treatment with amphotericin B was instituted for 30 days with satisfactory clinical response, recovery of joint mobility and improvement of pain. The patient was discharged using itraconazole, with good clinical and laboratory response.

CONCLUSION

The high prevalence in the world, the recent epidemic due to zoonotic transmission in Brazil and the different therapeutic approaches in view of the different clinical forms of the disease, demand that health professionals, both generalists and specialists, recognize the atypical manifestations of sporotrichosis. The treatment of lymphocutaneous and localized cutaneous forms tends to be done on an outpatient basis with itraconazole and the extracutaneous and disseminated cutaneous forms may require hospitalization and amphotericin B. In our case, we have osteomyelitis of atypical location in the interphalangeal areas with satisfactory response. The authors declare that the patient was oriented and signed the written informed consent form. 